\title{
Assessment of the relationship between psychological locus of control and immune status in breast cancer patients
}

\author{
Eman M. EL-Baiomy ${ }^{1}$, Mohamed L. Salem², Azza M. EL-Amir ${ }^{3}$, Noha A. Sabry and Nehal EL-Mashad ${ }^{5}$ \\ ${ }^{1}$ Immunology Department, Faculty of Science, Cairo University, Egypt \\ 2Zoology Department, Faculty of Science, Tanta University, Tanta, Egypt \\ ${ }^{3}$ Immunology Division, Faculty of Science, Cairo University, Egypt \\ ${ }^{4}$ Psychiatry Department, Faculty of Medicine, Cairo University, Egypt \\ ${ }^{5}$ Oncology Department, Faculty of Medicine, Tanta university, Egypt
}

Background: Several recent studies confirm the immune disturbances in cancer patients in comparison with the healthy individual. Other studies confirmed the effective role of psychological status on the immune system. In breast cancer patient, depression associated with decreased inflammatory and immune function. Several studies suggested relationship between locus of control and depression as an external locus of control individuals have more symptoms of depression. Aim: To investigate the direct relation between immune status and locus of control in breast cancer patients. Materials and Methods: Patients and participators responded to three questionnaires (God health locus of control, multidimensional health locus of control, and Rotter internal/external locus of control). The numbers and phenotype of CD4+ T cells, Treg cells and NK cells by flow-cytometry were measured. The gene expression of pro-inflammatory cytokines was assessed by real time PCR. Results: There is a significant direct co-relation between IHLC and CD4+ T cells, IHLC and NK cells. Conclusion: There is a relationship between psychological locus of control and immune status in breast cancer patients.

Keywords: Breast cancer; Immune status; Locus of control 